\title{
Distributed Central Pattern Generator Model for Robotics Application Based on Phase Sensitivity Analysis
}

\author{
Jonas Buchli and Auke Jan Ijspeert \\ Biologically Inspired Robotics Group \\ LSL, Swiss Federal Institute of Technology (EPFL), Lausanne \\ jonas.buchli@epfl.ch, auke.ijspeert@epfl.ch \\ http://birg.epfl.ch
}

\begin{abstract}
A method is presented to predict phase relationships between coupled phase oscillators. As an illustration of how the method can be applied, a distributed Central Pattern Generator (CPG) model based on amplitude controlled phase oscillators is presented. Representative results of numerical integration of the CPG model are presented to illustrate its excellent properties in terms of transition speeds, robustness and independence on initial conditions. A particularly interesting feature of the CPG is the possibility to switch between different stable gaits by varying a single parameter. These characteristics make the CPG model an interesting solution for the decentralized control of multilegged robots. The approach is discussed in the more general framework of coupled nonlinear systems, and design tools for nonlinear distributed control schemes applicable to Information Technology and Robotics.
\end{abstract}

\section{Introduction}

Information Technology has seen an unprecedented growth in possibilities and capacity in the second half of the 20th century. Powerful theories have emerged along with engineering principles that turn these theories into successful real world applications. Almost all of this progress has been made by adopting a linear and sequential approach to analyze and design systems. Under this view, each of the subsystems must be carefully engineered, in order to make them as reliable as possible. When connecting them, one is striving for a linear interaction as this allows one to guarantee that the prediction made for interacting subsystems remains valid. The order of operation, tasks and information flow is usually sequential as this simplifies the understanding of the mode of operation of the system and the identification of possible problems.

This is in contrast to how natural systems work. In nature, the subsystems are usually unreliable, non-uniform, noisy but in huge numbers. The subsystems and their interaction are of active, nonlinear nature, leading to emergent phenomena on the system level. Therefore, these systems often work naturally in a parallel fashion. This tends to give interesting properties to natural systems such as 
robustness, fast computation, high energy efficiency and versatility despite slow, noisy and unreliable components. In order to be able to construct systems with similar properties, it is crucial to have adequate theoretical tools for modeling and designing these complex systems.

In this article, we would like to contribute to this effort in the field of oscillatory systems. We develop a method for predicting phase relationships in systems of coupled oscillators, and use it to design systems that can switch between welldefined phase-locked states. In particular, we apply our approach to a concrete example: the distributed control of locomotion in robots with multiple degrees of freedom.

\section{Designing Biologically Inspired Distributed Controllers for Walking Robots}

Controlling walking in robots has proved to be a difficult engineering challenge. It requires coordinating multiple degrees of freedom using signals of the right frequencies, phases, and amplitude. As nature presents very robust and elegant solutions to that problem, some engineers have turned to biology as a source of inspiration. At first sight, the animal locomotory system seems to be of enormous complexity. But, despite the large number of elements taking part in locomotion control, a few simple common features have been observed by biologists among a large variety of different species. One of these is the notion of the Central Pattern Generator (CPG) [5,6]. A CPG is a network of neurons, capable of producing oscillatory signals without oscillatory inputs. For locomotion, CPGs are located in the spine, and receive relatively simple signals from higher centers of the brain for the control of speed and direction. Sensory feedback is usually not needed to produce the basic patterns, although it plays an important role in adapting the patterns to the given situation the animal is faced with.

Another important concept is to classify different walking patterns by the phase relationships between the individual limbs. This method allows to uncover striking similarities between the gait patterns observed in very different animals. In quadruped locomotion there are three gait patterns that are very often observed: walk, trot and bound.

Models of different complexity and based on different assumptions have been devised that can produce the abstract gait patterns [1,3,19-21]. One important approach is - motivated by the oscillatory limb movements - to use the most simple mathematical model that produces stable oscillatory behavior as gait pattern generator for one limb. This mathematical model is a nonlinear oscillator of some form. This oscillators are then connected together in order to achieve inter-limb coordination (see $[1,3,20]$ ).

Except $[20,21]$ most previous models use nonlinear oscillators that are motivated by neuronal circuits and that have therefore limit cycles with irregular shapes. In this contribution, the point is made to use the simplest oscillators possible as canonical subsystems, in order to have systems that are well understood and are simpler to treat analytically. The canonical subsystem is taken 
out of the class of nonlinear oscillators. In this article, the canonical subsystem which serves as model for the pattern generator of a single limb will be a simple amplitude controlled phase oscillator (ACPO). By this choice of the canonical subsystem one does avoid the problems involved with the aforementioned neuronal oscillators. The analytical treatment leads to a understanding of the system behavior that allows to apply synthetic approaches to construct a network with these canonical subsystems with desired global behavior. Furthermore, the network is constructed to have one single parameter by which the exhibited gait pattern can be controlled. This is a simplification comparing to previous approaches which usually need several parameters to be changed at the same time.

The desired properties that our CPG model should exhibit are the following. First, the CPG model should be independent of initial conditions and robust against perturbations. Second, the expressed gaits should ideally be controlled by one simple control variable. This simplifies control, and also replicates the biological observation that the modulation of a simple electrical stimulation signal is sufficient to change gait in cats [18]. Finally, when changing the control variable, the CPG should exhibit fast transitions, ideally within one cycle. The transitions are a critical moment since the animal can loose its stability if the transitions are not appropriate. Furthermore, fast transitions are also observed in nature. To the best of our knowledge, hitherto there exists no simple model that fulfills all the criteria just stated.

\section{Outline}

A short outline of our approach will be given as follows. First, the canonical subsystem will be presented. The notion of the phase will be introduced, since the phase is crucial to understand synchronization behavior. Then, it will be shown, that by examining the form of the limit cycle the sensitivity of the phase on perturbations can be derived. With this result, it will be shown how the phase relationship between two unidirectionally coupled oscillators can be derived. Out of the insights gained by that treatment, a method is presented to chose an arbitrary phase relationship between the two oscillators.

Next, a quadruped walking controller composed of four coupled oscillators will be constructed. The additional couplings give raise to additional constraints on the phase relationships. It will be shown by numerical experiments that only phase relationships that fulfill these constraints are stable. In a next step, it will be shown how we can exploit these additional constraints to have an continuous valued parameter that allows us to chose the gait pattern expressed. In the discussion we show how the results presented in this article fit in the larger picture and show that our assumptions and simplifications are based on firm theoretical grounds. 


\section{A Distributed Quadruped Central Pattern Generator}

\subsection{Predicting the Phase Between Two Oscillators}

Our goal in this section is to predict how an oscillator reacts to perturbations by looking at its limit cycle from a geometrical point of view, and to use this prediction for determining the phase relationship between coupled oscillators.

To start with the concepts needed to discuss nonlinear oscillators, the notion of a perturbed nonlinear dynamical system is introduced:

$$
\dot{\mathbf{q}}=F(\mathbf{q})+\mathbf{p}
$$

where $\mathbf{q}$ is the vector of state variables and $\mathbf{p}$ a perturbation vector. In the case the unperturbed system $(\mathbf{p}=0)$ converges to a periodic solution, it is called an oscillator and the set of $\mathbf{q}$ on which it continues to evolve is called the limit cycle of the system. As described in [15], every oscillator can be transformed into a phase $(\theta)$ - radius $(\mathbf{r})$ coordinate system:

$$
\begin{gathered}
\dot{\theta}=\omega_{0}+p_{\theta} \\
\dot{\mathbf{r}}=F_{r}(\mathbf{r}, \theta)+\mathbf{p}_{\mathbf{r}}
\end{gathered}
$$

where $\omega_{0}$ is the natural frequency of the (unperturbed) oscillator, $F_{r}$ is the dynamical system describing the evolution of $\mathbf{r}, p_{\theta}$ is the component of the perturbation acting on the phase and $\mathbf{p}_{\mathbf{r}}$ is the component of the perturbation acting in direction of the radius. Perturbations on a stable limit cycle have different effects on the phase depending on the $p_{\theta}$ and $\mathbf{p}_{\mathbf{r}}$ components. The $p_{\theta}$ component will modify the phase, since the phase is marginally stable [15]. On the other hand, the $\mathbf{p}_{\mathbf{r}}$ component, i.e. in the direction of the radius, will be damped out and will have little effect on the phase.

When two oscillators $\left(F_{1}, F_{2}\right.$ with corresponding state vectors $\left.\mathbf{q}_{\mathbf{1}}, \mathbf{q}_{\mathbf{2}}\right)$ are coupled together $\left(p_{\theta, 2}=f\left(\mathbf{q}_{\mathbf{1}}\right)\right)$, several types of dynamics can result including chaos (i.e. no periodic behavior) and phase-coupling. In this article, we are interested in 1:1 phase-locked regimes, i.e. when the oscillators synchronize such that $[15]$

$$
\theta_{d} \equiv \theta_{2}-\theta_{1} \approx \text { const }
$$

Assuming that the system has phase-coupled ${ }^{1}$, we are now interested in how to predict $\theta_{d}$ given two oscillators and their coupling. The general outline of our method is as follows:

1. From the limit cycle of the perturbed oscillator a sensitivity function $S_{p}(\mathbf{p})$ is derived.

2. From the limit cycle of the perturbing system (the other oscillator), the coupling and the sensitivity function, the perturbation term $p_{\theta}$ is calculated. $p_{\theta}$ is usually a function of the phase difference and the phase of the perturbed system.

\footnotetext{
${ }^{1}$ Determining which conditions are necessary for phase-coupling is out of the scope of the current article.
} 
3. From the requirement of phase synchronization (4) a differential equation (DE) for the phase difference between the perturbed and the perturbing system $\left(\theta_{d}\right)$ can be derived. This DE is usually a function of the phase difference and the phase of the perturbed system.

4. By integrating $p_{\theta}$ over the evolution of the perturbed limit cycle the perturbation of the phase that stays in the system is computed. This allows to derive a DE that only depends on $\theta_{d}$. By help of that DE, the fixed points for $\theta_{d}$ can be found.

5. By applying a stability analysis of the DE, the stable and unstable fixed points can be distinguished.

When looking at the phase space representation of a nonlinear dynamical system we can conclude that changes in the derivative of phase can only stem from components of the perturbation that are in direction of $\theta$, i.e. tangential to the limit cycle. The unit vector tangential to the limit cycle is

$$
\mathbf{e}_{\theta}=\frac{\dot{\mathbf{q}}}{|\dot{\mathbf{q}}|}
$$

Therefore, the effective perturbation on the phase is

$$
p_{\theta}=\mathbf{p} \cdot \mathbf{e}_{\theta}
$$

The derivative of the phase becomes

$$
\dot{\theta}=\omega_{0}+\mathbf{p} \cdot \mathbf{e}_{\theta}
$$

So we found the sensitivity of the phase on perturbations:

$$
S_{p}(\mathbf{p}) \doteq \frac{p_{\theta}}{|\mathbf{p}|}=\frac{\mathbf{p}}{|\mathbf{p}|} \cdot \mathbf{e}_{\theta}=\frac{\mathbf{p}}{|\mathbf{p}|} \cdot \frac{\dot{\mathbf{q}}}{|\dot{\mathbf{q}}|}
$$

With (7) we found an explicit form for the time evolution of $\theta$. By using the definition in (4) we can derive a differential equation for $\theta_{d}$. We require synchronization after some transient phase which is not discussed here:

$$
\int_{t_{1}}^{t_{2}} \dot{\theta}_{d} \mathrm{~d} t=0
$$

On the other hand

$$
\begin{gathered}
\dot{\theta}_{d}=\dot{\theta}_{2}-\dot{\theta}_{1} \\
\dot{\theta}_{d}=\omega_{0,2}+p_{\theta_{2}}-\left(\omega_{0,1}+p_{\theta_{1}}\right)
\end{gathered}
$$

This is usually a function of $\theta_{d}$ and $\theta_{2}$. As we are mainly interested in the steady state of the system, we integrate it over time. The integration over time is done implicitly by integration over $\theta_{2}$ (which increases monotonically with time), after the system reached the steady phase locked state. We assume the criterion for phase locking to be fulfilled at $\theta_{2}=\Theta_{0}$, and that the system subsequently is in the steady phase locked state for $\theta_{2}>\Theta_{0}$. From (9) we see that the integral should be zero

$$
\dot{\theta}_{d, \text { res }}=\lim _{\Theta \rightarrow \infty} \int_{\Theta_{0}}^{\Theta} \dot{\theta}_{d} \mathrm{~d} \theta_{2}=\sum_{n=1}^{\infty} \int_{2(n-1) \pi+\Theta_{0}}^{2 n \pi+\Theta_{0}} \dot{\theta}_{d} \mathrm{~d} \theta_{2} \equiv 0
$$


We now have outlined all the steps needed to arrive with a differential equation for $\theta_{d}$. In the following, we will show the analysis of the phase oscillator that will be used to construct the CPG.

\subsection{The Amplitude Controlled Phase Oscillator}

As outlined before, the CPG model will be constructed of simple canonical subsystems. In this case the subsystems are an amplitude controlled phase oscillator (ACPO). The ACPO is defined by the following dynamical system:

$$
[\dot{\theta}, \dot{r}]^{T}=\left[\omega,-g\left(r-r_{0}\right)\right]^{T}
$$

The description of this system can be transformed into an equivalent description in the Cartesian coordinate system $(x=r \cos \theta, y=r \sin \theta)$ :

$$
\dot{\mathbf{q}}=\left[\begin{array}{c}
\dot{x} \\
\dot{y}
\end{array}\right]=\left[g\left(\frac{r_{0}}{\sqrt{x^{2}+y^{2}}}-1\right) x-y \omega, g\left(\frac{r_{0}}{\sqrt{x^{2}+y^{2}}}-1\right) y+x \omega\right]^{T}
$$

A short hand notation of this system is introduced: $\dot{\mathbf{q}}=F_{\mathrm{ACPO}}(\mathbf{q})$, where $\mathbf{q}=$ $[x, y]^{T}$ is the state vector of the system. This system shows a limit cycle that has the form of a perfect circle with radius $r_{0}$ (Fig. 1(a)). The intrinsic frequency of the oscillator is $\omega$.

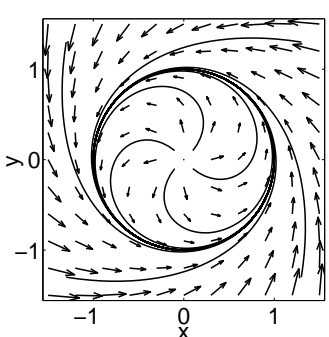

(a)

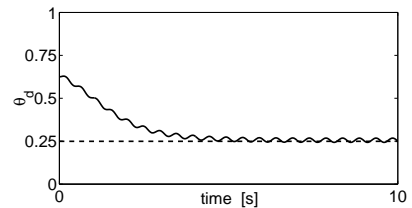

(b)

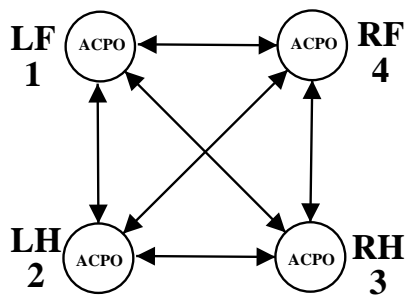

(c)

Fig. 1. a) Limit cycle of the amplitude controlled phase oscillator for $r_{0}=1, g=$ $10, \omega=2 \pi\left[\mathrm{rad} s^{-1}\right]$. The arrows show the flow $\dot{\mathbf{q}}$ defined by the $\left.F_{\mathrm{ACPO}}(13) . \mathbf{b}\right)$ This figure shows the phase difference established for the following values of $\omega_{d}=-0.0042$, $\lambda=2$ and $g=1000$. With help of (23) predicted value is $\theta_{d}=0.2493$ (dashed line). The value from numerical integration is shown with the solid line (mean over $t=[10,20]$ is $\left.\theta_{d}=0.2554\right)$. c) The structure of the ACPO-CPG. Note that the connections illustrated by arrows involve rotation matrices (compare to text).

\subsection{Two Coupled ACPO}

We introduce now a system of two ACPO where one ACPO is coupled unidirectionally to the other one.

$$
\begin{aligned}
& \dot{\mathbf{q}}_{1}=F_{\mathrm{ACPO}}\left(\mathbf{q}_{1}\right) \\
& \dot{\mathbf{q}}_{2}=F_{\mathrm{ACPO}}\left(\mathbf{q}_{\mathbf{2}}\right)+\mathbf{p}_{\mathbf{c}}\left(\mathbf{q}_{\mathbf{1}}\right)
\end{aligned}
$$


where $q_{1}=\left[x_{1}, y_{1}\right], q_{2}=\left[x_{2}, y_{2}\right]$. Next will be shown, how we can derive the phase relationship $\theta_{d}$ from the knowledge of the shape of the limit cycle and $\mathbf{p}_{\mathbf{c}}$. We will do this in an analytical way to illustrate how the method works. However, the method is not limited to cases where we know the form of the limit cycle by analytical derivation, but also works for cases where we get the form of the limit cycle and $f_{c}$ by numerical integration. To illustrate how the method works, consider the simple connection scheme:

$$
\mathbf{p}_{\mathbf{c}}=\lambda\left[0, x_{1}\right]^{T}
$$

In words: State variable $x$ from ACPO 1 is coupled on the derivative of state $y$ of ACPO 2 with a coupling constant $\lambda$.

1. We derive

$$
\mathbf{e}_{\theta_{\mathbf{2}}}=\frac{\dot{\mathbf{q}}_{\mathbf{2}}}{\left|\dot{\mathbf{q}}_{\mathbf{2}}\right|}=\left[-\sin \left(\theta_{2}\right), \cos \left(\theta_{2}\right)\right]^{T}
$$

2 .

$$
\mathbf{p}_{\mathbf{c}}=\lambda\left[0, x_{1}\right]^{T}=\lambda\left[0, r \cos \left(\theta_{1}\right)\right]^{T}
$$

From (6) we get

$$
p_{\theta_{2}}=\lambda r\left[0, \cos \left(\theta_{1}\right)\right]^{T} \cdot\left[-\sin \left(\theta_{2}\right), \cos \left(\theta_{2}\right)\right]^{T}=\lambda r \frac{1}{2}\left[\cos \left(\theta_{d}\right)+\cos \left(2 \theta_{2}-\theta_{d}\right)\right]
$$

3. Using (10) and (20)

$$
\dot{\theta}_{d}=\omega_{0,2}-\omega_{0,1}+\lambda r \frac{1}{2}\left[\cos \left(\theta_{d}\right)+\cos \left(2 \theta_{2}-\theta_{d}\right)\right]
$$

4. From (12) and (21) we get

$$
\begin{aligned}
\dot{\theta}_{d, r e s} & =\int_{0}^{2 \pi} \dot{\theta}_{d} \mathrm{~d} \theta_{2}=\int_{0}^{2 \pi}[\underbrace{\omega_{0,2}-\omega_{0,1}}_{\omega_{d}}+\lambda r \frac{1}{2}[\underbrace{\cos \left(\theta_{d}\right)}_{\text {const }}+\underbrace{\cos \left(2 \theta_{2}-\theta_{d}\right)}_{\text {periodic, zero mean }}]] \mathrm{d} \theta_{2} \\
& =2 \pi \omega_{d}+\lambda r \pi \cos \left(\theta_{d}\right) \equiv 0
\end{aligned}
$$

From this equation we can calculate the (averaged) fixed points for $\theta_{d}$

$$
\left.\theta_{d}\right|_{\dot{\theta}_{d, r e s} \equiv 0}=\arccos \left(-\frac{2 \omega_{d}}{\lambda r}\right)
$$

We note that we need $\left|\frac{2 \omega_{d}}{\lambda r}\right|<1$ for this particular system to phase-lock (i.e. for (23) to have equilibrium points). Since we assume steady phase locked state, $r \approx r_{0}$ can be assumed. We are interested in the stable fixed points, since they determine to which phase relationship the system will evolve. For example, for $\omega_{d}=0$, we find solutions at $\frac{\pi}{2}+n \pi, n \in \mathbb{Z}_{0}$.

5. The stability of the fixed points is determined by the one-dimensional Jacobian for $\theta$ which can be obtained by differentiating (22)

$$
\frac{\partial \dot{\theta}_{d}}{\partial \theta_{d}}=-\lambda r \pi \sin \left(\theta_{d}\right)
$$


From this equation we can calculate that $\frac{\partial \dot{\theta}_{d}}{\partial \theta_{d}}=-\lambda r<0$ for $\theta_{d}=\frac{\pi}{2}+2 n \pi$ and $\frac{\partial \dot{\theta}_{d}}{\partial \theta_{d}}=\lambda r>0$ for $\frac{3 \pi}{2}+2 n$ (for $\lambda>0$, opposite if $\lambda<0$ ). Therefore, for $\omega_{d}=0$ only phase differences $\theta_{d}=\frac{\pi}{2}+2 n \pi$ are stable fixed points.

Using (23) we can therefore determine the phase difference to which the two oscillators evolve when coupled, under the assumption that they phase-lock. For $\omega_{d} \neq 0$ the fixed points for $\theta_{d}$ have slightly different values and are dependent on the choice of $r_{0}$, as can be seen from (23). In Fig. 1(b) the results for numerical integration of the system treated above are presented for $\omega_{d} \neq 0$ and compared to the value predicted by the analytical treatment.

\subsection{Method for Choosing Arbitrary $\theta_{d}$}

Based on the insight gained in the previous section a method will be presented to chose arbitrary $\theta_{d}$. Therefore, a more general coupling scheme is introduced:

$$
\mathbf{p}_{2}=\lambda \mathbf{P} \mathbf{q}_{1}
$$

where $\mathbf{P}$ is the coupling matrix. In the aforementioned example (17) it would be

$$
\mathbf{P}=\left(\begin{array}{ll}
0 & 0 \\
1 & 0
\end{array}\right)
$$

We define a rotation matrix

$$
\mathbf{R}=\left(\begin{array}{cc}
\cos \theta_{R} & -\sin \theta_{R} \\
\sin \theta_{R} & \cos \theta_{R}
\end{array}\right)
$$

By taking $\mathbf{q}_{\mathbf{1}, \mathbf{r}}=\mathbf{R} \mathbf{q}_{\mathbf{1}}$, we get a vector that is equivalent to the vector $\mathbf{q}_{\mathbf{1}}\left(\theta_{1}^{\prime}\right)$, $\theta_{1}^{\prime}=\theta_{1}+\theta_{r}$. In other words, if we take $\mathbf{q}_{\mathbf{1}, \mathbf{r}}$ to perturb the second oscillator the effect is the same as if the first oscillator would be in state $\theta_{1}^{\prime}$. Thus,

$$
\mathbf{p}_{\mathbf{2}}=\lambda \mathbf{P} \mathbf{q}_{\mathbf{1}, \mathbf{r}}=\lambda \mathbf{P} \mathbf{R q}_{\mathbf{1}}=\lambda r\left[0, \cos \theta_{1} \cos \theta_{R}-\sin \theta_{1} \sin \theta_{R}\right]^{T}
$$

Using the same approach as in (20)-(23) we get

$$
\dot{\theta}_{d, r e s}=2 \pi \omega_{d}+\lambda r \pi\left[\cos \theta_{d} \cos \theta_{R}-\sin \theta_{d} \sin \theta_{R}\right] \equiv 0
$$

By exploiting the trigonometric addition theorems this transforms into

$$
\left.\theta_{d}\right|_{\dot{\theta}_{d, r e s} \equiv 0}=\arccos \left(-\frac{2 \omega_{d}}{\lambda r}\right)-\theta_{R}
$$

where again $r \approx r_{0}$ is the steady state behavior. As can be seen $\theta_{d}$ is directly proportional to the rotation angle $\theta_{R}$. Using (30) we can design couplings between the oscillators such as to obtain arbitrary phase difference between them. Note that the coupling does not need to be unidirectional. It is straight forward to introduce bidirectional coupling by changing (15) to

$$
\dot{\mathbf{q}}_{1}=F_{\mathrm{ACPO}}\left(\mathbf{q}_{1}\right)+\mathbf{p}_{\mathbf{c}}\left(\mathbf{q}_{2}\right)
$$

and working out the math as outlined above. Equivalently to (28), a second rotation matrix $\mathbf{P}_{\mathbf{2}}$ is introduced. Therefore, a third additive term in (29) arises. 


\subsection{The ACPO CPG}

The three most common gaits observed in quadrupeds are walk, trot and bound. To ease the notation, the legs of the quadruped are numbered in the following way: left front 1 , left hind 2 , right hind 3 , right front 4 (cf. Fig. 1(c)). If we define $\theta_{d, i j}=\theta_{i}-\theta_{j}$ as the difference between the phase of limb $i$ and $j$ then, the gaits can be classified according to Table 1(a) (the phases are normalized: $\theta=1$ corresponds to the full circle).

A quadruped CPG is constructed from four fully connected ACPO, i.e. all oscillators are coupled bidirectionally to every other one (see Fig. 1(c)). The coupling matrix is of the form

$$
\mathbf{P}=\left(\begin{array}{ll}
1 & 0 \\
0 & 1
\end{array}\right)
$$

and $\lambda=2$ for all connections. A ring structure basically is enough to build the $\mathrm{CPG}$, cf. $[1,16]$. However, the additional, redundant connections increase the speed of the gait transitions.

Let us outline how we can design specific gait patterns into this network. First of all, for this gait pattern the phase difference between the pairs of oscillators that are connected need to be known. We can derive these phase differences by help of Table 1(a). Then, for each connection a corresponding rotation matrix can be derived. If we take as an example the walk pattern we see that we come up with four different rotation matrices $\left(\theta_{d}= \pm 0.25 \pm 0.5\right)$ for the 12 connections. In order to be able to change from one gait pattern to another we make the rotation matrices dependent on a parameter $P_{\text {gait }}$ and we exploit the fact that $\mathbf{R}\left(\theta_{d}\right)=$ $\mathbf{R}^{-1}\left(-\theta_{d}\right)$. By analysis of the requirements needed to generate walk, trot and bound we come up with three parameter sets of $\theta_{R}$ (cf. Table 1(b)). Instead

Table 1. (a) The table shows the phase differences corresponding to the three most common gaits observed in quadrupeds. (b) The table shows the 3 different rotation angles that are needed in the construction of the ACPO-CPG. (c) Connection scheme used for the ACPO-CPG.

\begin{tabular}{|l||r|r|r|l||r|r|r|}
\hline & $\theta_{d, 12}$ & $\theta_{d, 13}$ & $\theta_{d, 14}$ \\
\hline walk & 0.75 & 0.25 & 0.5 \\
trot & 0.5 & 0.0 & 0.5 \\
bound & 0.5 & 0.5 & 0.0 \\
\hline
\end{tabular}

\begin{tabular}{|c|c|c|c|c|}
\hline & $\begin{array}{r}\mathrm{c} \\
\mathrm{LF} /\end{array}$ & $\begin{array}{l}\text { onnec } \\
\mathrm{LH} /\end{array}$ & $\begin{array}{l}\text { ion o } \\
\mathrm{RH}\end{array}$ & $\begin{array}{l}\operatorname{gin} \\
\mathrm{RF} / 4\end{array}$ \\
\hline $\mathrm{LF} / 1$ & 0 & $\mathbf{R}_{1}^{-1}$ & $\mathbf{R}_{2}$ & $\mathbf{R}_{3}^{-1}$ \\
\hline LH/2 & $\mathbf{R}_{1}$ & 0 & $\mathbf{R}_{3}^{-1}$ & $\mathbf{R}_{2}^{-1}$ \\
\hline 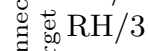 & $\mathbf{R}_{2}^{-1}$ & $\mathbf{R}_{3}$ & $0^{\circ}$ & $\mathbf{R}_{1}$ \\
\hline ठే ఫే $\mathrm{RF} / 4$ & $\mathbf{R}_{3}$ & $\mathbf{R}_{2}$ & $\mathrm{R}_{1}^{-1}$ & 0 \\
\hline
\end{tabular}

of fixing the $\theta_{d}$ we can define continuous functions that provide these values when a parameter $P_{\text {gait }}$ is increased. We chose the functions given in 33-35. This allows to chose the gait pattern by the single continuous valued parameter $P_{\text {gait }}$. The three corresponding rotation matrices are used in the connection scheme as presented in Table 1(c) (using the short notation $\mathbf{R}_{\mathbf{i}}=\mathbf{R}\left(\theta_{R, i}\right)$ ). 


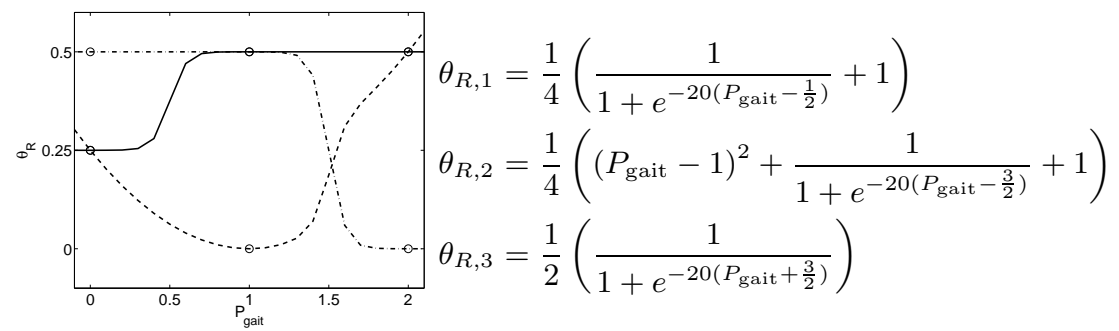

Fig. 2. $\theta_{R, 1,2,3}$ as a function of the chosen gait parameter. $P_{\text {gait }}=0$ corresponds to the walk pattern, $P_{\text {gait }}=1$ to trot, and $P_{\text {gait }}=2$ to the bound. Solid line: $\theta_{R, 1}$, dashed line: $\theta_{R, 2}$, dash-dotted line: $\theta_{R, 3}$. The dots correspond to values that correspond exactly to the values for the different gait patterns. However, also for settings quite far from these points the gait patterns are stable.

\subsection{Simulation Results of the ACPO-CPG}

In the following, the results of numerical integration of the ACPO-CPG are presented. The system was integrated with a variable step Runge-Kutta solver [11]. The tolerance settings were $T_{\text {rel }}=10^{-3}$ and $T_{\text {abs }}=10^{-6}$. The initial conditions were always chosen randomly in $\theta_{1,2,3,4} \in[-1,1]$. Because the system is robust against random initial conditions, we do not present the transient behavior at the beginning of the integration procedure but rather focus on the more interesting phenomena during gait transitions. In Fig. 3, all possible transitions are shown. The time $t=0$ always corresponds to the time when the gait control parameter $P_{\text {gait }}$ is changed abruptly from one setting to another. Noteworthy here is that not all transitions are made with the same ease. Especially the transitions from walk to bound and back take up to about $1.5 \mathrm{~s}$ to begin. Also the transient time is higher for these transitions. Furthermore, we have an asymmetry in transitions. The transitions from walk to bound is faster then from bound to walk. Interestingly, Kelso et al. [9] have shown the same effects when human subjects are asked to consciously switch from one coordination task to another. The authors also establish the link to the physical theories of complex systems that will be addressed in the discussion.

Random fluctuations play a very important role in synergetic systems. It turns out that they are fundamental to any pattern formation process. Furthermore, we want our model to be robust against noise. Therefore, we use a noisy model to test the influences of noise. For that the differential equation of the system gets transformed into a stochastic difference equation

$$
\Delta \mathbf{q}=(1+\xi) F(\mathbf{q}) \Delta t
$$

where $\xi$ is a uniformly distributed random number in $[-0.1,0.1]$. The stochastic difference equation was then integrated using the Euler method with a time-step of $\Delta t=10^{-4} \mathrm{~s}$. Representatively, for the illustration of the effect of the noise, the transition from bound to walk has been chosen, because from the above presented results it is known to be slowest. In Fig. 4(a) the results are presented 
a)

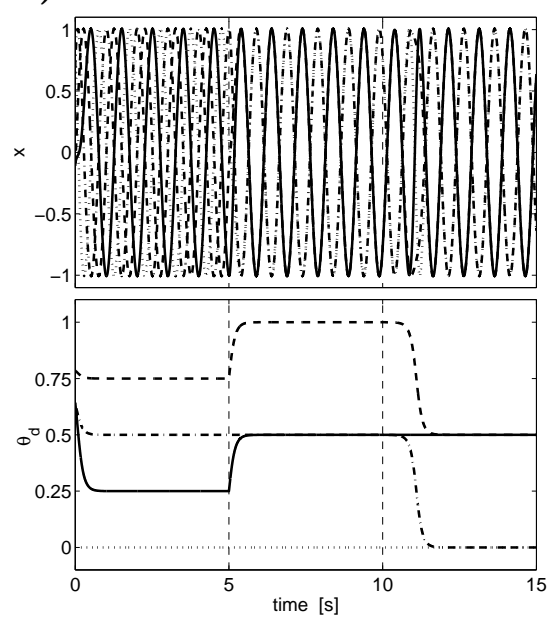

c)

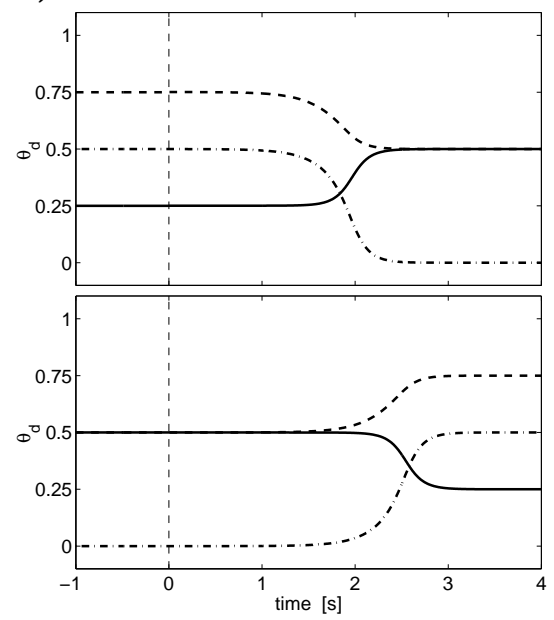

b)

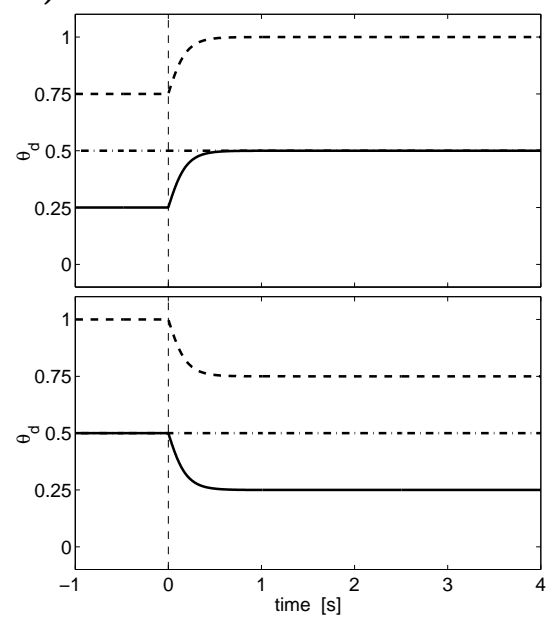

d)

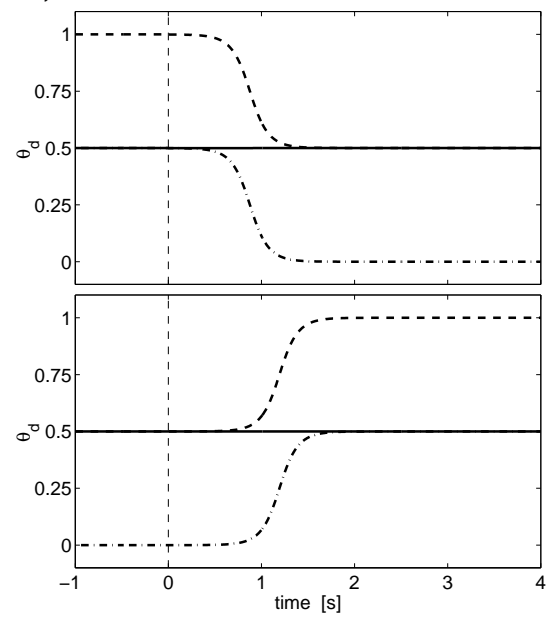

Fig. 3. Results of the numerical integration of the ACPO CPG. a) Trajectories of the ACPO-CPG when switching from walk to trot to bound and the corresponding phase difference plots $\left(\theta_{d, i j}\right)$. Dashed line: $\theta_{d, 12}$, solid line: $\theta_{d, 13}$, dash-dotted line: $\theta_{d, 14}$. The upper figure presents the oscillatory activity $\left(x_{i}\right)$, while the lower figure shows the corresponding phase difference evolution. b) phase difference plots for walk to trot (upper figure) and trot to walk (lower figure). The dashed vertical line indicates the time at which $P_{\text {gait }}$ is changed. c) walk to bound and bound to walk $\mathbf{d}$ ) trot to bound and bound to trot. 
and as can be seen the begin of the transition occurs about one second earlier, while the steady states are basically not affected by that noise level. Thus, our system is not only robust against noise, but even benefits from it. Such noise induced improvements has been shown in a variety of systems [4] and are now commonly called stochastic resonance.

Finally, in order to illustrate one significant advantage of dynamical systems based CPG models for controlling walking over other methods (e.g. trajectory replay), we present the behavior of the model in case of an external disturbance in Figs. 4(b) and 4(c).

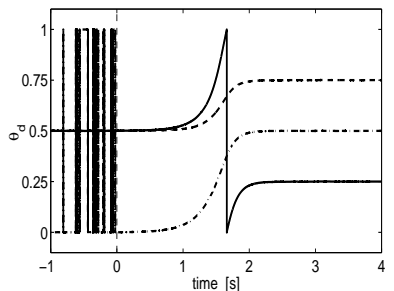

(a)

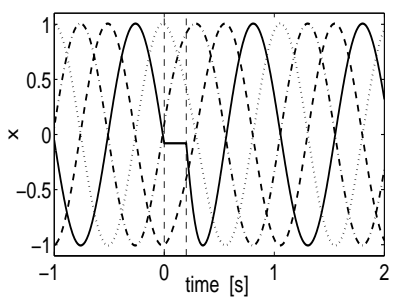

(b)

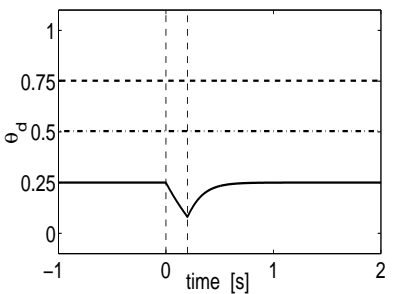

(c)

Fig. 4. a) Further experiments on the influence of perturbations on the transition speed. Representatively the bound to walk transitions is chosen which is the slowest. Noise is added during the integration procedure (see text). As can be observed the transition is initiated about $1 \mathrm{~s}$ earlier then in the case without noise. b), c) To illustrate the robustness against perturbation that is inherently built in the structurally stable dynamical system model of the CPG we present the case when the state variable for the left hind leg gets fixed for $0.2 \mathrm{~s}$ and then released again during walk. The two vertical lines show the time when the legs is fixed and released again. As can be observed, the leg increases in speed in order to catch up with the other legs to fulfill the requirements of the gait pattern. Within less than $0.5 \mathrm{~s}$, the normal gait is re-established.

\section{Discussion}

The ACPO CPG. We have presented a model for a quadruped central pattern generator. The model is of distributed nature and shows fast transitions and only one global attractor. It is robust against noise and perturbations. By one continuous variable we have the control over the chosen gait patterns. From the algorithmic point of view the model is very simple. Considering all these properties, we conclude that the ACPO-CPG is a viable candidate for the implementation in a robot. The presented CPG is however only applicable to interlimb coordination. Additional oscillators are needed for intralimb coordination (i.e. coordinating different DOFs at the hip, knee, and ankle). However, the presented methodology is applicable for these problems as well.

The choice of the subsystem in form of simple oscillators $[1,3,19]$, and more specifically phase oscillators $[20,21]$ has been presented before. However, we motivate our choice with concepts from physics of complex systems rather then base the model on simplified cell models. That this abstraction implied by the choice of simple oscillators makes sense and is based on firm theoretical grounds 
can be seen when looking on the observations made by biologists from a complex systems perspective.

Modeling in the complex systems framework. We argue that these observations (i.e. low dimensional dynamics and autonomous oscillatory behavior of nerve centers) and the resulting abstract concepts (i.e. CPG) are not a coincidence, but rather a necessity. The reason for that necessity can be understood by physical theories of complex systems developed over the last few decades. These theories deal with systems that are constructed from active subsystems. Understanding the concepts covered by these theories and gaining the insight that modeling controllers for walking robots is an example of a much broader class of problems, we can turn the physical theories into a design methodology that allows us to decide which features need to be preserved in our model and which one can be abstracted away in order to arrive with a controller that satisfies given global properties.

Haken [7] puts the argument forward that a large ensemble of interacting systems normally exhibits low-dimensional dynamics under very broad conditions. While others have formulated parts of the ideas before it was his contribution to formulate an integrated theory of such systems, which he called synergetic systems. He enhances the concept of the order-parameter introduced by Landau [10]. The order parameters are identified as slowly evolving variables in a dynamical system (e.g. in the laser, a prime example of self-organizing systems, the order-parameter is the field strength of the laser light). The order-parameters turn out to be the instable modes of that system and their number remains usually a very few comparing to the full state space of the system. The key point is that all the other variables of the system follow the order parameters, and, on the other hand, the activity of the full system influences the evolution of the order parameters. Haken formulated that fact in the slaving principle. One can build hierarchies of systems where the order parameters of one subsystem constitute the subsystems for the next hierarchy level. In the case of the locomotory system the order parameters of interest are the phase relationships between the limbs. The scales of the order parameters and the subsystems differ in about three orders of magnitude (neurons: $\sim 10^{-3} \mathrm{~s}-\operatorname{limb}$ activity $\sim 10^{0} \mathrm{~s}$ ). The different scales are typical for synergetic systems. Furthermore, it has been shown theoretically [7] and experimentally [14], that the behavior of the order parameters is very independent of the exact nature of the subsystems. Even more, at the order parameter level, completely new phenomena can occur, which are not foreseeable at the subsystem level ${ }^{2}$.

Conclusion of the complex systems perspective. Considering the aforementioned facts, it gets clear that there are two approaches of modeling the behavior of such systems. Both of them have strengths and weaknesses. The first method is to derive models for the subsystems and couple them to come up with the complete model. This is an important approach, especially if one is interested in the exact behavior of the real system being modeled and the influence of all the parameters (for an example see [13]). However, especially when the chosen

\footnotetext{
${ }^{2}$ aka. emergence, network effects, self-organization
} 
level of description is very detailed, this method is rather tedious, it leads to complicated models that are normally computationally intensive and possess a large number of parameters. One has to have an enormous knowledge of the details of the subsystems which in reality is often missing. Especially, if one is successful with this modeling approach, one will rediscover the aforementioned system hierarchies. The other approach is to focus on the order-parameter level, if one is mainly interested in mimicking the overall system behavior. It is an phenomenological approach. The advantage here, is that one is freed from a huge amount of parameters, the systems are usually simple and easy to simulate. Yet, the physics guarantees that we still catch the important aspect of the system behavior, namely the behavior of the order-parameters (i.e. models for human inter-limb coordination see $[8,9])$. The model derived by this approach typically consists of one low-dimensional dynamical system describing the behavior of the order parameters (e.g. quadruped CPG see [16]). In this article we are following the second approach.

As we are free to chose which level of the system hierarchy we would like to model in order to arrive with an usable model for a robotic application, a good approach is to keep a distributed model consisting of a few subsystems. The subsystems themselves are still models of complex systems. Therefore, they model order-parameter behavior. Naturally, one splits up the whole system into subsystems, where the system being modeled also shows some modularization (i.e. Body segments, Limbs, ...) or where we identify parts that lend themselves to easy measurement of the subsystem behavior. In case of the walking controller, the order parameters are the population activity of the motoneurons for one limb. The population activity serves to drive the muscles. The subsystems are the single neurons of the limb CPG, the muscle cells and all the other numerous parts that form the neuro-mechanical system. Because we are at the order-parameter level of description, it gets clear that there is no need to use models that are motivated by observations made on the single neuron in order to model the behavior on the CPG level. Another motivation for the choice of the canonical subsystem in form of a simple phase oscillator is the fact that from an mathematical viewpoint all limit cycle systems belong to the same universality class [2]. I.e. effects observed in one limit cycle system are also to be expected in another limit cycle system (However, in practical cases, the relationship is often enough only accessible in a qualitative manner. Even, if, from the mathematical viewpoint, a quantitative relationship exists). Furthermore, with this method we arrive with a model that does not show certain drawbacks of earlier models such as dependence on initial conditions, slow and lacking transitions, or periodic driving and prove therefore that our modeling approach is viable. Most probably the most fundamental advantage for our goal of controlling robots is that by choosing the simple oscillator model, we can predict the phase relationships with more ease and to a certain extent by analytical methods.

The level of abstraction of the ACPO-CPG corresponds to the order parameter description of dynamical systems. At this level of description a very simple model can be derived as shown by [16]. The model presented by Schöner et al. 
however is not of distributed nature anymore. As mentioned before, one interesting property of synergetic systems is their distributed nature. In a robot one would like to have simple distributed control for low level tasks such has locomotion, thus allowing a central processor to use its power to address more involved tasks, such as path planning, communication and the like. Therefore, in this contribution we constructed a model with a more complex structure, that lends itself for a distributed implementation in a robot built of uniform elements.

Outlook and future work. From a more theoretical point of view it will be interesting to do more rigorous analysis of the model, e.g. bifurcation analysis. Furthermore, it will be interesting to take a closer look at the improvement by noise, and compare the observation to other examples and theoretical considerations about stochastic resonance. It is known that there exists a certain optimal level of noise for a given system. This optimum remains to be found.

Since the characteristics of coupled dynamical systems, that we exploited to construct the ACPO-CPG, are universal characteristics that can be observed in many real world systems such as semiconductors [14], analog electronics [12], chemical reactions [17] and many more, one is basically able to implement this models on top of a variety of substrates. The choice in nature are neurons, but for applications we are not restricted to this substrate. The substrate of choice for implementation in the long term will be the one where we have the appropriate control over the characteristic time and length scales on one hand, and suitable operation conditions (temperature, field strengths, power consumption) on the other hand. In addition to that, it should be cheap and simple to manufacture. Therefore, to find such suitable substrates and the way of implementing the systems on top of them, a lot of experiments have to be done.

Conclusions. In recent years a lot of progress has been made in understanding complex systems from a theoretic point of view. Moreover, advances in technology allows us to implement and partially simulate systems of a complexity hitherto impossible. Yet, for applications, these powerful concepts are not yet exploited in a systematic fashion. Researchers in different fields often make implicit use of the concepts contained in the theory of complex systems when they make investigations and observations, yet, sometimes make assumptions that are not well aligned with this theory. In the authors opinion, it is important and one of the grand challenges for the next decades to transform the knowledge into design principles and collect experiences in order to harness the full power of active distributed systems. The research presented here, belongs to a more general effort that aims at using theories of coupled dynamical systems in the solution of difficult engineering problems and tries to devise new design principles. The possible fields of application are numerous - network engineering, multichannel information transmission, sensor networks and robotics just to name a few.

Acknowledgments This research is funded by a Young Professorship Award to Auke Ijspeert from the Swiss National Science Foundation. We would like to thank Jun Nakanishi for constructive comments on an earlier version of this article. 


\section{References}

1. J.J. Collins and S.A. Richmond. Hard-wired central pattern generators for quadrupedal locomotion. Biological Cybernetics, 71(5):375-385, 1994.

2. M.J. Feigenbaum. Universal behavior in nonlinear systems. Los Alamos Science, $1(1): 4-27,1980$.

3. Y. Fukuoka, H. Kimura, and A.H. Cohen. Adaptive dynamic walking of a quadruped robot on irregular terrain based on biological concepts. The International Journal of Robotics Research, 3-4:187-202, 2003.

4. L. Gammaitoni, P. Hänggi, P. Jung, and F. Marchesoni. Stochastic resonance. Reviews of Modern Physics, 70(1):223-287, 1998.

5. S. Grillner. Control of locomotion in bipeds, tetrapods and fish. In V.B. Brooks, editor, Handbook of Physiology, The Nervous System, 2, Motor Control, pages 1179-1236. American Physiology Society, Bethesda, 1981.

6. S. Grillner. Neurobiological bases of rhythmic motor acts in vertebrates. Science, 228(4696):143-149, 1985.

7. H. Haken. Advanced Synergetics. Instability Hierarchies of Self-Organization Systems and Devices., volume 20 of Springer Series in Synergetics. Springer Verlag Berlin Heidelberg, 2nd edition, 1987.

8. J. A. S. Kelso, J. P. Scholz, and G. Schöner. Nonequilibrium phase transitions in coordinated biological motion: critical fluctuations. Physics Letters A, 118(6):279$284,1986$.

9. J. A. S. Kelso, J. P. Scholz, and G. Schöner. Dynamics governs switching among patterns of coordination in biological movement. Physics Letters A, 134(1):8-12, 1988.

10. L.D. Landau and E.M. Lifshitz. Statistical Physics, volume 5 of Course of theoretical physics. Pergamon Press London Paris, 1959.

11. The MathWorks Inc. Matlab web site. http://www.mathworks.com.

12. V.I. Nekorkin and M.G. Velarde, editors. Synergetic Phenomena in Active Latices, volume 79 of Springer Series in Synergetics. Springer Verlag Heidelberg, 2002.

13. D. Noble. Modeling the heart - from genes to cells to the whole organ. Science, 295:1678-1682, 2002.

14. J. Peinke, J. Parisi, O.E. Rössler, and R. Stoop. Encounter with Chaos. SelfOrganized Hierarchical Complexity in Semiconductor Experiments. Springer Verlag Berlin Heidelberg, 1992.

15. A. Pikovsky, R. Rosenblum, and J. Kurths. Synchronization, A universal concept in nonlinear sciences, volume 12 of Cambridge Nonlinear Science Series. Cambridge University Press, Cambridge, UK, 2001.

16. G. Schöner, W.Y. Jiang, and J.A.S. Kelso. A synergetic theory of quadrupedal gaits and gait transitions. Journal of theoretical Biology, 142:359-391, 1990.

17. I. Schreiber and M. Marek. Modeling the Dynamics of Biological Systems, volume 65 of Springer Series in Synergetics, chapter Dynamics of Oscillatory Chemical Systems. Springer Verlag Berlin Heidelberg, 1995.

18. M.L. Shik, F.V. Severin, and G.N. Orlovsky. Control of walking by means of electrical stimulation of the mid-brain. Biophysics, 11:756-765, 1966.

19. G. Taga. A model of the neuro-musculo-skeletal system for human locomotion. I. Emergence of basic gait. Biological Cybernetics, 73(2):97-111, 1995.

20. K. Tsuchiya, S. Aoi, and K. Tsujita. Locomotion control of a multi-legged locomotion robot using oscillators. In 2002 IEEE Intl. Conf. SMC, volume 4, 2002.

21. H. Yuasa and M. Ito. Coordination of many oscillators and generation of locomotory patterns. Biological Cybernetics, 63:177-184, 1990. 